\title{
PLASMA LIPOPROTEIN PROFILES AND ARYLESTERASE ACTIVITIES IN TWO INBRED STRAINS OF RABBITS WITH HIGH OR LOW RESPONSE OF PLASMA CHOLESTEROL TO DIETARY CHOLESTEROL
}

\author{
A. C. Beynen, ${ }^{*} \dagger$ M. B. Katan $\ddagger$ and L. F. M. VAN ZutPhen* \\ *Department of Laboratory Animal Science, State University, P.O. Box 80.166, 3508 TD Utrecht, The \\ Netherlands (Tel: 030-532033); and \\ $\ddagger$ Department of Human Nutrition, Agricultural University, De Dreijen 12, $6703 \mathrm{BC}$ Wageningen, The \\ Netherlands (Tel: 08370-82589)
}

(Received 22 March 1984)

\begin{abstract}
Cholesterol feeding for 4 weeks of female and male rabbits of two inbred strains increased plasma cholesterol concentrations by about 11 and $48 \mathrm{mmole} / \mathrm{l}$ in the hypo- and hyperresponsive strain, respectively.

2. On the low-cholesterol pre-experimental diet, the hyporesponsive animals had significantly higher plasma HDL (high density protein) cholesterol levels than hyperresponders.

3. In both strains, cholesterol feeding caused elevations of cholesterol in all lipoprotein classes, the difference between the hypo- and hyperresponsive strains in essence only being observed in the VLDL (very low density lipoprotein) fraction.

4. Basal plasma total arylesterase activity was significantly higher in the hypo- than in the hyperresponsive rabbits.

5. Dietary cholesterol caused an increase in plasma esterase activity in both strains.

6. We suggest that in rabbits a low plasma arylesterase activity and a low concentration of HDL cholesterol are associated with an increased sensitivity to dietary cholesterol.
\end{abstract}

\section{INTRODUCTION}

Between animals of the same species there can be marked differences in plasma cholesterol responses to an increased intake of cholesterol. Certain individuals (hyporesponders) show only small changes in the concentration of cholesterol in the plasma, whereas others (hyperresponders) develop high degrees of hypercholesterolaemia. This phenomenon has been well established in monkeys (Clarkson et al., 1971; Eggen, 1976), rabbits (Roberts et al., 1974; Van Zutphen and Fox, 1977) and rats (Imai and Matsumura, 1973; Van Zutphen and Den Bieman, 1981). Human hypo- and hyperresponders to dietary cholesterol also appear to exist (Katan and Beynen, 1983). Inbred strains of rabbits (Van Zutphen and Fox, 1977) and rats (Van Zutphen and Den Bieman, 1981) with either a high or low cholesterolaemic response to high-cholesterol diets have been identified.

In the present study we have used male and female animals of both a hypo- and hyperresponsive inbred strain of rabbits. Two questions were addressed. First, we wanted to know whether the distribution of cholesterol between the plasma lipoproteins both before and after cholesterol feeding were different between the two strains. In a recent study we have shown that the pre-experimental lipoprotein profile and the effect of dietary cholesterol on this profile varies markedly between animal species (Terpstra and Beynen, 1984).

$†$ To whom correspondence should be addressed.
Secondly, we determined plasma total arylesterase activities. We have recently demonstrated that basal plasma esterase activity was significantly higher in an inbred rat strain which is hyporesponsive to dietary cholesterol than in a hyperresponsive strain. Furthermore, we found that plasma esterase activity was increased after feeding the rats a high-cholesterol diet (Beynen et al., 1984). These data suggest that arylesterases are associated with cholesterol metabolism and with the sensitivity to dietary cholesterol. We now investigated whether these observations in rats extend to hypo- and hyperresponsive rabbit strains.

\section{MATERIALS AND METHODS}

\section{Animals and diets}

Male and female rabbits of two inbred strains were used The strains were IIIVO/Ju and $\mathrm{AX} / \mathrm{Ju}$ and originated from the Jackson Laboratory colony, Bar Harbor, ME, USA (Fox, 1975). The strains had previously been shown to be hypo- and hyperresponsive to dietary cholesterol (Van Zutphen and Fox, 1977). The animals are maintained at the Department of Laboratory Animal Science and kept individually in cages with wire mesh bases constructed of galvanized steel in a room with controlled lighting (14 hr/day), constant temperature $\left(16-20^{\circ} \mathrm{C}\right)$ and humidity.

During the pre-experimental period, the rabbits were fed commercial rabbit pellets (LK04, Hope Farms, Woerden, The Netherlands). Food and water were provided ad libitum. As of Day 0 of the experiment the diet was fortified with $0.5 \%$ cholesterol; the animals received this diet for 28 days. The commercial and high-cholesterol diet contained $(\mathrm{g} / 100 \mathrm{~g})$ : moisture, 8.1 and 9.3 ; ash, 8.3 and 8.1 ; crude 
protein, 17.7 and 17.7 ; crude fat, 3.5 and 3.7 , respectively The cholesterol content of the diets as measured by gasliquid chromatography (Nordby and Nagy, 1973) of the non-saponifiable fraction, was 7 and $521 \mathrm{mg} / 100 \mathrm{~g}$, respectively. The high-cholesterol diet was provided on a restricted basis, the rabbits receiving $90 \mathrm{~g} / \mathrm{day}$. Most rabbits consumed all their food within $4 \mathrm{hr}$. Water was provided ad libitum.

Sampling of blood and analytical methods

Samples of blood were taken from the marginal ear vein of the rabbits into heparinized tubes. Sampling was performed between 08.00 and $10.00 \mathrm{hr}$ after the removal of any remaining food at $16.00 \mathrm{hr}$ the previous day. Cholesterol in plasma was measured enzymatically (Röschlau et al., 1974) using the kit (Monotest) supplied by Boehringer-Mannheim $\mathrm{GmbH}, \mathrm{FRG}$. The coefficient of variation was routinely less than $1 \%$.

The method of Terpstra et al. (1981) was used for separating lipoproteins by ultracentrifugation. Plasma samples were stored at $4^{\circ} \mathrm{C}$ for periods of up to two days. Cholesterol in the lipoprotein fractions was determined as described above. Recovery of lipoprotein-cholesterol (plasma total cholesterol $=100 \%$ ) was $116 \pm 12$ and $82 \pm 15$ (mean $\pm \mathrm{SD}, n=12$ ) for the low- and high-cholestero period, respectively.

Plasma total arylesterase activities were determined at pH 8.6 using $\beta$-naphthylpropionate as a substrate according to Pilz (1961). Reaction conditions were chosen so that the amount of product formed was linear with time and enzyme concentration. Enzyme activity was corrected for spontaneous hydrolysis of the substrate. Plasma samples were stored at $-20^{\circ} \mathrm{C}$ for 5 weeks until analysis. All samples were analysed within one batch; the coefficient of variation was less than $1.5 \%$

\section{RESULTS}

Table 1 confirms (cf. Van Zutphen and Fox, 1977) that the $\mathrm{AX} / \mathrm{J}$ and IIIVO/J rabbit strains are hyper-

*Abbreviations: VLDL, very low density lipoproteins; IDL, intermediate density lipoproteins; LDL, low density lipoproteins; HDL, high density lipoproteins. and hyporesponsive to dietary cholesterol with regard to the level of plasma cholesterol. In addition, we now show that this holds not only for the males but also for the females. After 4 weeks of cholesterol feeding plasma cholesterol levels were about 4-fold higher in the hyper- than in the hyporesponders. The hyporesponsive females tended to be somewhat less sensitive than the hyporesponsive males; such a sex difference was not seen for the hyperresponders ( $\mathrm{Ta}$ ble 1). The initial plasma cholesterol values were significantly increased in the hyperresponsive females when compared with the other groups. Body weights throughout the experiment were similar for all experimental groups (Table 1).

The lipoprotein profiles of the individual animals before and after cholesterol feeding can be seen from the photograph of the pre-stained plasma after density gradient ultracentrifugation (Fig. 1). The lipoprotein profiles before the dietary challenge show VLDL* (density $(d)<1.006 \mathrm{~g} / \mathrm{ml}$ ) at the top of the tube, in most cases an LDL $(1.019<d<1.063)$ and HDL band $(1.063<d<1.125)$ further down, and residual stain at the base. There was a striking difference between the basal density profiles of female hypo- and hyperresponders. The hyporesponsive females showed a heavily stained HDL band, which was not seen in the hyperresponders (Fig. 1). The hyperresponding females however, displayed an intensively stained LDL band. A more pronounced HDL band was also observed in the male hyporesponders when compared with the hyperresponders. With respect to the LDL band, no difference between male hypo- and hyperresponders could be detected. The initial lipoprotein profiles of the male hyporesponders showed some interindividual variation (Fig. 1).

Four weeks after cholesterol feeding there was no difference between the lipoprotein profiles of hypoand hyperresponsive rabbits. Based on the intensity

Table 1. Effect of dietary cholesterol on body weight and plasma cholesterol levels in hypo- and hyperresponsive rabbits



Results are expressed as means \pm SD for three animals in each group. On Day 0 of the experimen all animals were switched from the commercial to the high-cholesterol diet. 'Day 0 ; ${ }^{2}$ Day 28 *Significantly different from hyporesponders $(P<0.05$; two-tailed Student's $t$-test). 


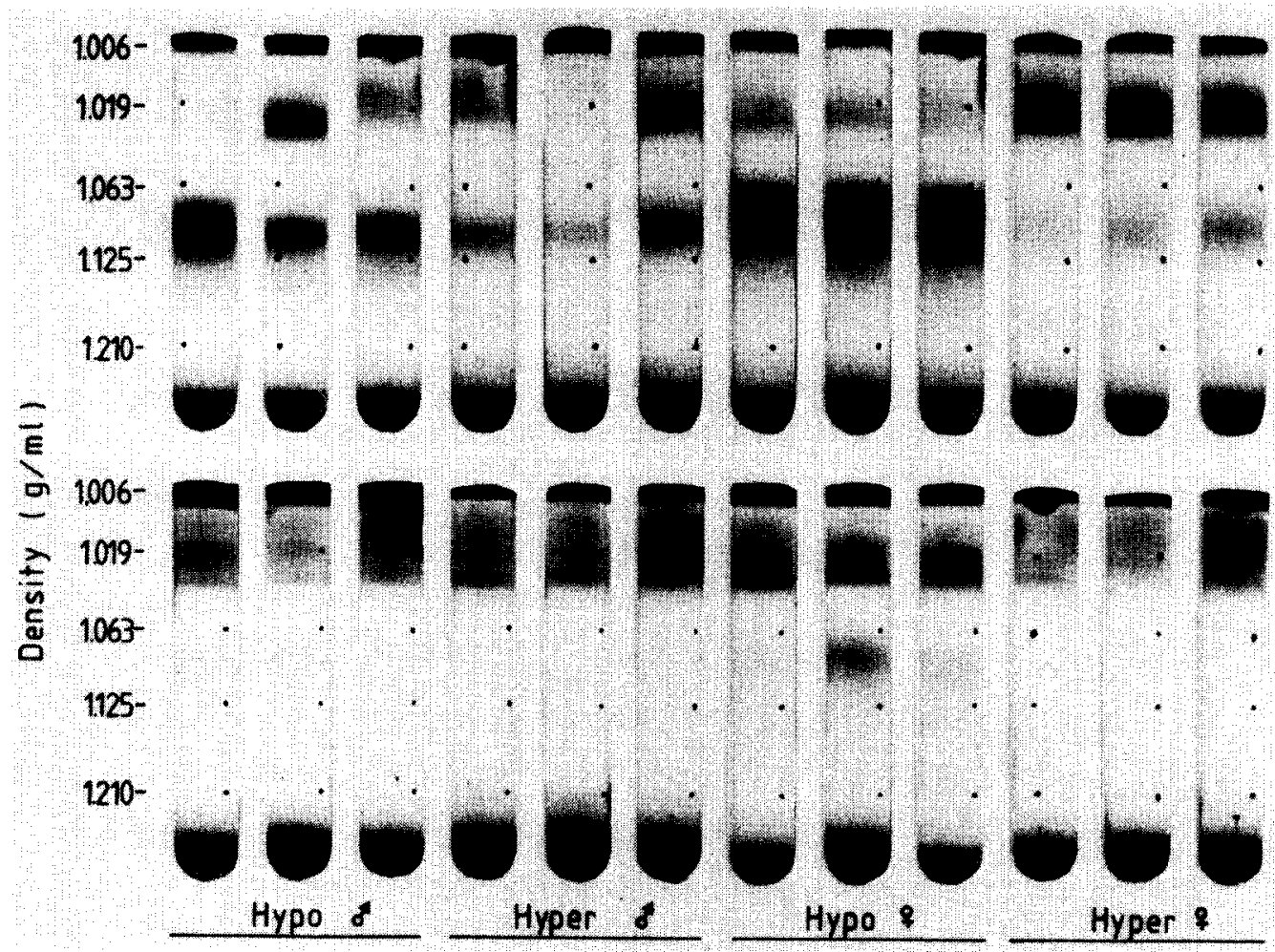

Fig. 1. Photograph of the density profile of Sudan-black stained lipoproteins observed after ultracentrifugation of plasma from individual rabbits before (top row) and 4 weeks after (bottom row) cholesterol feeding.

of the Sudan black staining, it would appear that dietary cholesterol induces a decrease in the HDL fraction and an increase in lipoproteins within the LDL and IDL $(1.006<d<1.063)$ density range.

Table 2 documents the cholesterol concentrations of plasma lipoproteins in the rabbits. As would be anticipated on the basis of Fig. 1, before cholesterol feeding the hyporesponsive females had higher concentrations of cholesterol in the $\mathrm{HDL}_{2}$ fraction $(1.063<d<1.125)$ and lower concentrations in the LDL fraction when compared with the hyperresponsive females (Table 2). With regard to the $\mathrm{HDL}_{2}$ fraction unlike the LDL fraction, this difference persisted when lipoprotein cholesterol was expressed as percentage of total plasma cholesterol. On the low-cholesterol commercial diet, the hyporesponsive females carried $64 \%$ of their plasma cholesterol in the $\mathrm{HDL}_{2}$ fraction and $20 \%$ in the $\mathrm{LDL}$ fraction; for the hyperresponding females these values were 14 and $25 \%$, respectively. The VLDL- and IDL-cholesterol concentrations in the hyperresponding females were significantly higher than in their hyporesponsive counterparts. The proportions of VLDL cholesterol were 34 and $13 \%$, and the proportions of IDL cholesterol were 25 and $11 \%$ in the hyper- and hyporesponders. In the males, a difference between hypo- and hyperresponders while on the low-cholesterol diet was only seen for $\mathrm{HDL}_{2}$ cholesterol, the former animals having about twice as much. When expressed relative to total plasma cholesterol, $45 \%$ of cholesterol was located in the $\mathrm{HDL}_{2}$ fraction in the hyporesponders and $28 \%$ in the hyperresponders.
After 4 weeks of cholesterol feeding, cholesterol had increased in all lipoprotein fractions. Most of the extra cholesterol in the plasma was carried by the VLDL fraction. In essence, the hypo- and hyperresponders only differed in their VLDL-cholesterol concentrations. In the males, the hyperresponders also had significantly higher LDL-cholesterol levels (Table 2).

Plasma esterase activities were not significantly different between male and female rabbits (Table 3 ). The initial esterase activity was significantly lower in the hyperresponsive strain. Cholesterol feeding increased plasma esterase activities in both strains, the activity in the hyporesponsive rabbits remaining higher than in the hyperresponders (Table 3).

\section{DISCUSSION}

The two inbred strains of rabbits $(\mathrm{AX} / \mathrm{Ju}$ and IIIVO/Ju) differed markedly in their response of plasma cholesterol to dietary cholesterol. This confirms our earlier work with male rabbits (Van Zutphen and Fox, 1977), and shows further that this strain difference also holds for female animals. In both strains the excess of cholesterol in the plasma after cholesterol feeding was mainly located in the VLDL fraction, the increase in VLDL cholesterol being 2.5-fold higher in the male and 10-fold higher in the female hyperresponders than in their hyporesponsive counterparts. In the other lipoprotein fractions there also was an increase in the concentration of cholesterol, but the changes were rather similar in hypo- and hyperresponders (Table 2). The 
Table 2. Effect of dietary cholesterol on the distribution of cholesterol between plasma lipoproteins in hypo- and hyperresponsive rabbits

\begin{tabular}{|c|c|c|c|c|c|c|c|}
\hline \multirow{3}{*}{\multicolumn{2}{|c|}{$\begin{array}{l}\text { Lipoprotein } \\
\text { fraction }\end{array}$}} & \multicolumn{6}{|c|}{ Lipoprotein cholesterol (mmol/l of whole plasma) } \\
\hline & & \multicolumn{3}{|c|}{ Males } & \multicolumn{3}{|c|}{ Females } \\
\hline & & нуро & & Hyper & нуро & & Hyper \\
\hline \multirow[t]{2}{*}{ VLDL } & initial & $0.17 \pm$ & 0.02 & $0.36 \pm 0.06 \star$ & 0.13 & \pm 0.06 & $0.88 \pm 0.41 *$ \\
\hline & final & $9.82 \pm$ & 2.46 & $25.08 \pm 5.48^{\star}$ & 2.91 & \pm 1.72 & $31.53 \pm 4.67 *$ \\
\hline \multirow[t]{2}{*}{ IDL } & initial & $0.13 \pm$ & 0.05 & $0.12 \pm 0.06$ & 0.11 & \pm 0.04 & $0.65 \pm 0.05 *$ \\
\hline & final & $1.97 \pm$ & 0.20 & $1.73 \pm 0.59$ & 1.51 & \pm 0.60 & $1.96 \pm 0.69$ \\
\hline \multirow[t]{2}{*}{ LDL } & initial & $0.20 \pm$ & 0.13 & $0.12 \pm 0.07$ & 0.20 & \pm 0.07 & $0.64 \pm 0.17 *$ \\
\hline & final & $2.45 \pm$ & 0.37 & $4.24 \pm 0.50 \star$ & 2.28 & \pm 0.87 & $3.85 \pm 0.89$ \\
\hline \multirow[t]{2}{*}{$\mathrm{HDL}_{2}$} & initial & $0.45 \pm$ & 0.11 & $0.20 \pm 0.07 \star$ & 0.63 & \pm 0.11 & $0.36 \pm 0.05 *$ \\
\hline & final & $0.92 \pm$ & 0.17 & $1.04 \pm 0.15$ & 0.91 & \pm 0.16 & $0.97 \pm 0.14$ \\
\hline \multirow[t]{2}{*}{$\mathrm{HDL}_{3}$} & initial & $0.17 \pm$ & 0.02 & $0.08 \pm 0.01 *$ & 0.15 & \pm 0.03 & $0.10 \pm 0.01 *$ \\
\hline & final & $0.27 \pm$ & 0.05 & $0.27 \pm 0.17$ & 0.23 & \pm 0.03 & $0.39 \pm 0.09 *$ \\
\hline
\end{tabular}

Results are expressed as means $\pm \mathrm{SD}$ for three animals in each group. On Day 0 (initial) all animals were transferred to the high-cholesterol diet for another 28 days (final).

*Significantly different from hyporesponders $(P<0.05$; two-tailed Student's $t$-test). Density $(d)$ ranges of lipoproteins in $\mathrm{g} / \mathrm{ml}$ : VLDL, $d<1.006 ; \mathrm{IDL}, 1.006<d<1.019 ; \mathrm{LDL}, 1.019<d<1.063$; $\mathrm{HDL}_{2}$. $1.063<d<1.125 ; \mathrm{HDL}_{3}, 1.125<d<1.21$

increase in VLDL cholesterol probably reflects the accumulation of so-called $\beta$-VLDL particles. Dietary cholesterol has been shown to induce the formation of these particles in dogs, rats, rabbits and monkeys (Mahley, 1978). The $\beta$-VLDL particles contain large amounts of cholesteryl esters, arginine-rich protein (apoprotein E) and migrate on electrophoresis with $\beta$-lipoprotein mobility (Mahley, 1978).

The lipoprotein profile of Sudan-black stained lipoproteins was drastically changed by cholesterol feeding (Fig. 1). Based on the intensity of the Sudanblack staining, it would appear that HDL lipo- proteins had disappeared and that IDL lipoproteins had accumulated. Similar dietary-cholesterol-induced changes in the lipoprotein profile were seen earlier in lean Zucker rats and chickens (Terpstra and Beynen, 1984). The lipoprotein pattern in these cholesterol-fed animals resembles that found in type III hypercholesterolaemic patients (Terpstra and Beynen, 1984). It is noteworthy that the decrease in Sudanblack stained material in the $\mathrm{HDL}_{2}$ band of the cholesterol-fed rabbits (Fig. 1) was accompanied by an increase in $\mathrm{HDL}_{2}$ cholesterol (Table 2). This suggests that other changes in the $\mathrm{HDL}_{2}$ lipoproteins

Table 3. Effect of dietary cholesterol on plasma arylesterase activities in hypo- and hyperresponsive rabbits

\begin{tabular}{|c|c|c|c|c|c|c|c|}
\hline & \multicolumn{7}{|c|}{ Plasma arylesterase activity ( $\mathrm{mol} / \mathrm{min} / \mathrm{ml})$} \\
\hline & \multicolumn{3}{|c|}{ Males } & \multicolumn{3}{|c|}{ Females } & \\
\hline & Hypo & & Hyper & Hypo & & Hyper & \\
\hline initial & 17.38 & \pm 4.59 & $10.40 \pm 0.84 *$ & 21.86 & \pm 2.88 & $14.05 \pm$ & $\pm 1.44^{\star}$ \\
\hline Einal & 21.66 & \pm 1.40 & $14.63 \pm 0.34^{*}$ & 23.45 & \pm 2.67 & $16.10+$ & $\pm 0.68 *$ \\
\hline change & +4.27 & \pm 4.07 & $+4.23 \pm 1.09 \star \star$ & +1.59 & \pm 3.91 & $+2.05 \pm$ & $\pm 0.77 \star \star$ \\
\hline
\end{tabular}

Results are expressed as means + SD for three animals in each group. Values are given before (initial) and 4 weeks after (final) cholesterol feeding.

*Significantly different from hyporesponders; **significantly different from zero (two-tailed Student's $t$-test). 
are responsible for the decreased affinity for Sudan black. This is in line with earlier observations that the intensity of the colour of the lipoproteins in the gradient does not always give a good impression of the concentration of cholesterol in the lipoproteins (Terpstra and Beynen, 1984).

On the low-cholesterol commercial diet the hyporesponsive rabbits carried more cholesterol in the $\mathrm{HDL}_{2}$ fraction than the hyperresponders, and this was so both in absolute and relative terms. This would suggest that a high $\mathrm{HDL}_{2}$-cholesterol level is a predisposing factor for a low plasma cholesterol response to dietary cholesterol. This is in line with findings of Rudel et al. (1981) who found in randombred African green monkeys that the individual plasma cholesterol response to dietary cholesterol was negatively associated with the level of HDL cholesterol. On the other hand, in our studies with human volunteers we observed a positive relationship; the subjects with the highest serum cholesterol response to dietary cholesterol had the highest initial HDL cholesterol values (Katan and Beynen, 1984). The implications of these relationships and the discrepancy between humans and rabbits and monkeys are not clear at present.

Although the physiological function of plasma arylesterases is obscure, they may be involved in cholesterol metabolism. In inbred strains of rabbits (Van Zutphen and Fox, 1977) and rats (Okamoto et al., 1972; Van Zutphen and Den Bieman, 1981) it was found that the variation in the response of plasma cholesterol level to dietary cholesterol was associated with a genetically determined variation in plasma esterases. A fast moving esterase zone on starch-gel electrophoresis was present in the plasma of hyporesponsive strains, but not in that of hyperresponsive strains. This qualitative difference between hypo- and hyperresponders corresponds with the plasma total arylesterase activity. We found that the initial plasma esterase activity was significantly higher in an inbred rat strain which is hyporesponsive to dietary cholesterol than in a hyperresponsive strain (Beynen et al., 1984). We now observed that this also holds for two inbred rabbit strains with a high or low cholesterolaemic response to dietary cholesterol (Table 3 ). In individual random-bred rabbits we did not find an association between initial plasma total esterase activity and the subsequent plasma cholesterol response to cholesterol feeding (Beynen et al., 1984). This may be explained by the facts that random-bred rabbits vary considerably in their plasma-esterase zymograms (Van Zutphen, unpublished) and that rabbit plasma contains at least 12 different arylesterases (Pilz et al., 1968). On the other hand, only one of the esterases (Est-2) in the zymogram of rabbit plasma has been found to correlate with the plasma cholesterol response to dietary cholesterol (Van Zutphen and Fox, 1977). Thus it would be desirable to study and measure the plasma esterases separately.

Loading the rabbits with dietary cholesterol caused an increase in plasma total esterase activity (Table 3 ). Similar results were obtained earlier in rats (Beynen et al., 1984). It is possible that the increase in esterase activity results from a dietary-cholesterol-induced release of esterases from the intestine. In rats, Lewis and Hunter (1966) found a marked increase in ester- ases with higher electrophoretic mobility in the intestinal lymph, and later in the serum, following the intragastric injection of fat. Possibly, these esterases interact with dietary cholesterol at the level of the mucosal cell, while low esterase activities are accompanied by a reduced handling of dietary cholesterol resulting in accumulation in the plasma. More experimental work is needed to shed more light on the functions of plasma arylesterases. The use of inbred strains of animals with defined, but different plasma esterase patterns, will be of great importance in this respect.

Acknowledgements-We thank A. Versluis for taking care of the rabbits and Th.J. Bakker, A. Lankhorst and Z. Kruyswijk for expert analytical assistance. MBK is an established investigator of the Netherlands Heart Foundation.

\section{REFERENCES}

Beynen A. C., Weinans G. J. B. and Katan M. B. (1984) Arylesterase activities in the plasma of rats, rabbits and humans on low- and high-cholesterol diets. Comp. Biochem. Physiol. 78B, 669-673.

Clarkson T. B., Lofland H. B., Bullock B. C. and Goodman H. O. (1971) Genetic control of plasma cholesterol. Studies on squirrel monkeys. Archs Path. 92, 37-45.

Eggen D. A. (1976) Cholesterol metabolism in groups of rhesus monkeys with high or low response of serum cholesterol to an atherogenic diet. J. Lipid Res. 17, 663-673.

Fox R. R. (1975) Handbook on Genetically Standardized JAX Rabbits. Jackson Laboratory, Bar Harbor, Maine, USA.

Imai Y. and Matsumura H. (1973) Genetic studies on induced and spontaneous hypercholesterolemia in rats. Atherosclerosis 18, 59-64.

Katan M. B. and Beynen A. C. (1983) Hyper-response to dietary cholesterol in man. Lancet $\mathbf{I}, 1213$.

Katan M. B. and Beynen A. C. (1984) HDL cholesterol, LDL receptor activity and response to dietary cholesterol. Atherosclerosis (in press).

Lewis A. A. M. and Hunter R. L. (1966) The effect of fat ingestion on the esterase isozymes of intestine, intestinal lymph, and serum. J. Histochem. Cytochem. 14, 33-39.

Mahley R. W. (1978) Alterations in plasma lipoproteins induced by cholesterol feeding in animals including man. In Disturbances in Lipid and Lipoprotein Metabolism (Edited by Dietschy J. M., Gotto A. M. Jr and Ontko J. A.), pp. 181-197. American Physiological Society, Bethesda, USA.

Nordby H. E. and Nagy S. (1973) An evaluation of recent gas-liquid chromatographic liquid phases for resolution of acetylated plant sterols. J. Chromatogr. 75, 187-193.

Okamoto K., Yamori Y., Ooshima A. and Tanaka T. (1972) Development of substrains in spontaneously hypertensive rats: genealogy, isozymes and effect of hypercholesterolemic diet. Jap. Circ. J. 36, 461-470.

Pilz W. (1961) Methode zur photometrischen Mikrobestimmung kleiner Mengen $\beta$-Naphtol in Gegenwart eines grossen Ueberschusses verschiedener $\beta$-Naphtylester-Emulsionen und deren Verwendung zur Bestimmung der "Aromatischen Esterase" in biologischen Material. Mikrochem. Acta 1, 614-633.

Pilz W., Stelzl E. and Johann I. (1968) Untersuchungen über esterspaltende Enzyme von Versuchstieren und Methoden zu ihrer routinemässigen Bestimmung, IV. Die Arylesterasen des Kaninchenserums und ihre physiologische Funktion. Enzym. biol. clin. 9, 97-123.

Roberts D. C. K., West C. E., Redgrave T. G. and Smith J. B. (1974) Plasma cholesterol concentration in normal 
and cholesterol-fed rabbits. Its variation and heritability. Atherosclerosis 19, 369-380.

Röschlau P., Bernt E. and Gruber W. (1974) Enzymatische Bestimmung des Gesamt-Cholesterins im Serum. Z. klin. Chem. klin. Biochem. 12, 403-407.

Rudel L. L., Reynolds J. A. and Bullock B. C. (1981) Nutritional effects on blood lipid and HDL cholesterol concentrations in two subspecies of African green monkeys (Cercopithecus aethiops). J. Lipid Res. 22, 278-286.

Terpstra A. H. M. and Beynen A. C. (1984) Density profile and cholesterol concentration of serum lipoproteins in experimental animals and human subjects on hypercholesterolaemic diets. Comp. Biochem. Physiol. 77B, 523-528.
Terpstra A. H. M., Woodward C. J. H. and Sanchez-Muniz F. J. (1981) Improved techniques for the separation of serum lipoproteins by density gradient ultracentrifugation: visualization by prestaining and rapid separation of serum lipoproteins from small volumes of serum. Analyt. Biochem. 111, 149-157.

Van Zutphen L. F. M. and Den Bieman M. G. C. W. (1981 Cholesterol response in inbred strains of rats. Rattu: norvegicus. $J$. Nutr. 111, 1833-1838.

Van Zutphen L. F. M. and Fox R. R. (1977) Stratin differences in response 10 dietary cholesterol by JAX rabbits: correlation with esterase patterns. Atherosclerosis 28. $435-446$. 